\title{
NONLINEAR ELLIPTIC BOUNDARY VALUE PROBLEMS AND THE GENERALIZED TOPOLOGICAL DEGREE
}

\author{
BY FELIX E. BROWDER
}

Communicated March 9, 1970

Introduction. It is our purpose in the present note to present a general existence theorem for noncoercive elliptic boundary value problems for operators of the form:

$$
A(u)=\sum_{|\alpha| \leq m}(-1)^{|\alpha|} D^{\alpha} A_{\alpha}\left(x, u, \cdots, D^{m} u\right),
$$

on closed subspaces $V$ of the Sobolev space $W^{m, p}(G), G$ an open subset of $R^{n}, n \geqq 1$. This existence theorem is based upon an extension of the theory of the generalized topological degree for $A$-proper mappings of Banach spaces introduced in Browder-Petryshyn [8], [9], and, in particular, on an extension of the Borsuk-Ulam theorem to pseudomonotone mappings $T$ from a reflexive separable Banach space $V$ to its conjugate space $V^{*}$.

To make a precise statement of our general existence theorem possible, we introduce the following notation: For a given $m \geqq 1$, we let $\xi$ be the $m$-jet of a function $u$ from $R^{n}$ to $R^{s}$ for some given $s \geqq 1$, i.e. $\xi=\left\{\xi_{\alpha}:|\alpha| \leqq m\right\}$, and set

$$
\zeta=\left\{\zeta_{\alpha}:|\alpha|=m\right\}, \quad \eta=\left\{\eta_{\beta}:|\beta| \leqq m-1\right\},
$$

where each $\xi_{\alpha}, \zeta_{\alpha}$, and $\eta_{\beta}$ is an element of $R^{s}$. The set of all $\xi$ of the above form is an Euclidean space $R^{r_{m}}$, and correspondingly, $\zeta \in R^{r_{m}^{\prime}}$, $\eta \in R^{r_{m-1}}$.

For each $\alpha, A_{\alpha}$ is assumed to be a function from $G \times R^{r_{m}}$ to $R^{s}$ satisfying the following conditions:

Assumptions on $A(u):(1) A_{\alpha}(x, \xi)$ is measurable in $x$ for fixed $\xi$ and continuous in $\xi$ for fixed $x$. For a given $p$ with $1<p<\infty$, there exists a constant $c$ such that

$$
\left|A_{\alpha}(x, \xi)\right| \leqq c\left(\left(1+\sum_{|\beta| \leqq m}\left|\xi_{\beta}\right|^{p_{\alpha \beta}}\right)\right.
$$

with $p_{\alpha \beta} \leqq(p-1)$ for $|\alpha|=|\beta|=m$, and

AMS 1969 subject classifications. Primary 3547, 3536, 4780, 4785; Secondary 5536.

Key words and phrases. Nonlinear elliptic boundary value problems, generalized topological degree, Sobolev space, coercive, pseudomonotone, Borsuk-Ulam theorems, limit of $A$-proper mappings. 


$$
\begin{array}{r}
p_{\alpha \beta}<\frac{n p+p(m-|\alpha|)-n}{n-p(m-|\beta|)}, \quad \text { if } m-\frac{n}{p} \leqq|\alpha| \leqq m, \\
m-\frac{n}{p} \leqq|\beta| \leqq m, \\
|\beta|+|\alpha|<2 m, \\
p_{\alpha \beta} \leqq \frac{n p}{n-p(m-|\beta|)}, \quad \text { if }|\alpha|<m-\frac{n}{p}, \\
m-\frac{n}{p} \leqq|\beta| \leqq m .
\end{array}
$$

(2) If $\xi=(\zeta, \eta)$, then for each $x$ in $G, \eta$ in $R^{r_{m-1}}, \zeta$ and $\zeta^{\prime}$ in $R^{r_{m}}$ with $\zeta \neq \zeta^{\prime}$

$$
\sum_{|\alpha|=m}\left\langle A_{\alpha}(x, \zeta, \eta)-A_{\alpha}(x, \zeta, \eta), \zeta_{\alpha}-\zeta_{\alpha}^{\prime}\right\rangle>0,
$$

(where $\langle\cdot, \cdot\rangle$ denotes the inner product in $R^{s}$ ).

(3) For each $\gamma$ and $\gamma^{\prime}$ in $R^{r_{m}^{\prime}}$,

$$
\sum_{|\alpha|=m}\left\langle A_{\alpha}(x, \zeta, \eta)-\gamma_{\alpha}, \zeta_{\alpha}-\gamma_{\alpha}^{\prime}\right\rangle \rightarrow \infty \quad(|\zeta| \rightarrow \infty),
$$

uniformly for bounded $\eta$.

Let $W^{m, p}(G)$ be the Sobolev space of $s$-vector functions $u$ such that $u$ and all its derivatives $D^{\alpha} u$ for $|\alpha| \leqq m$ lie in $L^{p}(G)$ where $p$ is the exponent involved in the inequalities of Assumption (1). Then for any $u$ and $v$ in $W^{m, p}(G)$, we may define the generalized Dirichlet form corresponding to the representation (1) by:

$$
a(u, v)=\sum_{|\alpha| \leq m}\left(A_{\alpha}(\xi(u)), D^{\alpha} v\right),
$$

where

$$
\begin{array}{rlrl}
\xi(u) & =\left\{D^{\alpha} u:|\alpha| \leqq m\right\}, & & A_{\alpha}(\xi(u))(x)=A_{\alpha}(x, \xi(u)(x)), \\
(w, v) & =\int_{G}\langle w(x), u(x)\rangle d x, & & \text { (integration with respect to } \\
& \text { Lebesgue } n \text {-measure). }
\end{array}
$$

TheOREM 1. Let $G$ be an open subset of $R^{n}$ with $G$ bounded and the Sobolev Imbedding Theorem valid on $G$ (i.e. G satisfies mild smoothness conditions on its boundary). Let $A(u)$ be a quasilinear elliptic operator of order $2 m$ on $G$ of the form

$$
A(u)=\sum_{|\alpha| \leq m}(-1)^{|\alpha|} D^{\alpha} A_{\alpha}(\xi(u)),
$$


where the coefficient functions $A_{\alpha}$ satisfy Assumptions (1), (2), and (3) above. Suppose that $A(u)$ is odd in u, i.e. $A_{\alpha}(x,-\xi)=-A_{\alpha}(x, \xi)$ for each $\alpha$ and all $x$ in $G, \xi$ in $R^{r_{m}}$. For each $w$ in $V^{*}$, the dual space of a closed subspace $V$ of $W^{m, p}(G)$, consider the problem of finding $u$ in $V$ such that $a(u, v)=(w, v)$ for all $v$ in $V$. Suppose that there exists a continuous function $\phi: R^{+} \rightarrow R^{+}$such that for each solution $u$ of this problem for any $w$ in $V^{*}$,

$$
\|u\|_{V}=\|u\|_{W^{m, p}(G)} \leqq \phi\left(\|w\|_{\left.V^{*}\right)} .\right.
$$

Then for each w in $V^{*}$, there exists at least one solution $u$ in $V$ of the problem: $a(u, v)=(w, v)$ for all $v$ in $V$.

We have used the notation $(w, v)$ in Theorem 1 to denote the pairing between $w$ in $V^{*}$ and $u$ in $V$.

THEOREM 2. Let $G$ be a bounded, smoothly bounded open set in $R^{n}$ (as in Theorem 1), and consider a one-parameter family of operators $A_{t}(u), t \in[0,1]$, where for each $t$,

$$
A_{t}(u)=\sum_{|\alpha| \leq m}(-1)^{|\alpha|} D^{\alpha} A_{\alpha}(\xi(u) ; t)
$$

and the coefficient functions are continuous in $t$, uniformly for bounded $\xi$ and all $x$ outside a null set in $G$. For each $t$, we take the generalized Dirichlet form

$$
a_{t}(u, v)=\sum_{|\alpha| \leq m}\left(A_{\alpha}(\xi(u) ; t), D^{\alpha} v\right),
$$

where we assume that $A_{t}(u)$ satisfies Assumptions (1), (2), (3) for each $t$ in $[0,1]$. Suppose that $A_{1}(u)$ is odd, and that there exists a continuous function $\phi: R^{+} \rightarrow R^{+}$such that if $a_{t}(u, v)=(w, v)$ for some $w$ in $V^{*}, u$ in $V, t$ in $[0,1]$ and all $v$ in $V$, then $\|u\|_{V} \leqq \phi\left(\|w\|_{V^{*}}\right)$.

Then the problem: $a_{0}(u, v)=(w, v)$ for all $v$ in $V$; has a solution $u$ in $V$ for each w in $V^{*}$.

Theorem 2 includes Theorem 1 as the special case in which $A_{t}(u)$ $=A(u)$ for all $t$ in $[0,1]$. It also includes the standard existence theorem for $A(u)$ in which the Dirichlet form $a(u, v)$ is assumed to be coercive, i.e.

(6) There exists $c: R^{+} \rightarrow R^{1}$ with $c(r) \rightarrow \infty$ as $r \rightarrow \infty$ such that $a(u, u)$ $\geqq c\left(\|u\|_{V}\right)\|u\|_{V}$.

Indeed, if $A(u)$ is coercive, and if we set $A_{t}(u)=A(u)-t A(-u)$ for $t$ in $[0,1]$, then $A_{0}(u)=A(u), A_{1}(u)$ is odd, the Assumptions (1), (2), and (3) hold for every $A_{t}(u)$, while since $a_{t}(u, u)=a(u, u)-$ $t a(-u, u)=a(u, u)+t a(-u,-u)$, it follows that 


$$
a_{i}(u, u) \geqq(1+t) c\left(\|u\|_{v}\right)\|u\|_{v} \geqq c\left(\|u\|_{V}\right)\|u\|_{V}
$$

provided that $\|u\|_{V}>R$, where $c(r)>0$ for $r>R$. Suppose that for some $u$ in $V$, $w$ in $V^{*}$ and $t$ in $[0,1]$, we have

$$
a_{t}(u, v)=(w, v) \quad(v \in V) .
$$

Then:

$$
c\left(\|u\|_{v}\right)\|u\|_{V} \leqq a_{t}(u, u)=(w, u) \leqq\|w\|_{V *}\|u\|_{v},
$$

and as a consequence $c\left(\|u\|_{V}\right) \leqq\|w\|_{v^{*}}$ if $u=0$. If we set $\phi(s)=$ $\sup \{r: c(r) \leqq s\}$, it follows that $\|u\|_{v} \leqq \phi\left(\|w\|_{v^{*}}\right)$ and by Theorem 2, the equation $a(u, v)=(w, v)(v \in V)$, has a solution $u$ in $V$ for each $w$ in $V^{*}$.

Existence theorems for elliptic boundary problems of this type were first obtained by Višik [15] using compactness arguments and a priori estimates on $(m+1)$ st derivatives. Monotonicity arguments were first applied to these problems in Browder [2], [3], using the basic existence theorem for monotone maps from a reflexive Banach space $V$ to $V^{*}$ proved independently by Browder [2] and Minty [12]. The existence theorem in the coercive case was extended to elliptic operators $A(u)$ satisfying Assumptions (1), (2), and (3) by LerayLions [11]. Borsuk-Ulam theorems for monotone and semimonotone operators in infinite dimensional Banach spaces were first derived by Browder [4], [5], and were first applied to odd, homogeneous, elliptic operators satisfying strong monotonicity conditions by Pohožaev [14]. Theorem 1 was first obtained under a stronger hypothesis (3)' rather than (3) in Browder [6], together with Assumptions (1) and (2) on $A(u)$. This is as follows:

$(3)^{\prime}$ There exist continuous functions $k(\eta), k_{0}(\eta)>0$ such that

$$
\sum_{|\alpha| \leqq m}\left\langle A_{\alpha}(x, \zeta, \eta) \zeta_{\alpha}\right\rangle \geqq k_{0}(\eta)|\zeta|^{p}-k(\eta),
$$

for all $x$ in $G, \zeta$ in $R^{r_{m}^{\prime}}, \eta$ in $R^{r_{n-1}}$.

1. Proofs of Theorems 1 and 2 rest upon general results concerning two classes of nonlinear mappings of monotone type from a reflexive Banach space $V$ to its conjugate space $V^{*}$.

Definition 1. Let $V$ be a Banach space, $V^{*}$ its conjugate space, $T$ a mapping from $V$ to $V^{*}$, Then:

(a) $T$ is said to be pseudomonotone if for any sequence $\left\{u_{j}\right\}$ in $V$ with $u_{j}$ converging weakly to $u$ in $V$ such that $\lim \sup \left(T u_{j}, u_{j}-u\right) \leqq 0$, it follows that for any $v$ in $V$, $\lim \inf \left(T u_{j}, u_{j}-v\right) \geqq(T u, u-v)$.

(b) $T$ is said to satisfy condition $(S)_{+}$if for any sequence $u_{j}$ in $V$ with 
$\left\{u_{j}\right\}$ converging weakly to $u$ in $V$ for which $\lim \left(T u_{j}, u_{j}-u\right) \leqq 0$, it follows that $u_{j}$ converges strongly to $u$ in $V$.

Proposition 1. Suppose that A satisfies Assumption (1). Then there exists a continuous bounded mapping $T$ of $V$ into $V^{*}$ for a given closed subspace $V$ of $W^{m, p}(G)$ such that for all $u$ and $v$ of $V,(T u, v)=a(u, v)$. If $A(u)$ satisfies Assumptions (2) and (3), $T$ is pseudomonotone. If $A(u)$ satisfies Assumptions (2) and (3)', then $T$ satisfies condition $(S))_{+}$

The proof of Proposition 1 is given in $\$ 1$ of [7], and Appendix to $\$ 1$. Pseudomonotonicity was first defined by Brézis in [1] (though our definition differs slightly from his in considering only sequences rather than filters). The condition $(S)_{+}$was first defined in connection with the study of nonlinear eigenvalue problems in Browder [6] and is studied in detail in Browder [7], [8].

Theorem 3. Let $V$ be a reflexive separable Banach space, $T$ a mapping of $V$ into $V^{*}$ which is pseudomonotone, bounded on bounded sets, and continuous from each finite dimensional subspace of $V$ to the weak topology of $V^{*}$. Then:

(a) If $T$ is an odd mapping outside of some ball around the origin and if $T^{-1}(B)$ is bounded for any bounded subset $B$ of $V^{*}$, then $R(T)$, the range of $T$, is all of $V^{*}$.

(b) If $\left\{T_{t}\right\}$ is a family of bounded, pseudomonotone, finitely continuous mappings from $V$ to $V^{*}$ which is continuous in $t$ uniformly on bounded subsets of $V$, with $T_{0}=T, T_{1}$ odd outside some ball, and if there exists a function $\phi: R^{+} \rightarrow R^{+}$such that $T_{t}(u)=w$ implies that

$$
\|u\| \leqq \phi(\|w\|) \quad(t \in[0,1])
$$

then $R(T)=V^{*}$.

Theorem 3 and Proposition 1 together imply the validity of Theorems 1 and 2. Theorem 3 follows from an extension to the class of pseudomonotone mappings from $V$ to $V^{*}$ of the theory of the generalized degree defined for $A$-proper mappings of Banach spaces in Browder-Petryshyn [9], [10] and applied to mappings $T$ from a reflexive $V$ to $V^{*}$ satisfying condition $(S)$ in Chapter 17 of Browder [8]. The basic facts are summarized in the following theorem:

THEOREM 4. Let $V$ be a reflexive separable Banach space, $V^{*}$ its conjugate space. Let $T$ be a mapping from $V$ to $V^{*}$ which is finitely continuous from $V$ to $V^{*}$ (i.e. continuous from each finite dimensional subspace of $V$ to the weak topology of $V^{*}$ ) and bounded (i.e. maps bounded subsets of $V$ into bounded subsets of $\left.V^{*}\right)$. Then: 
(a) If $T$ is pseudomonotone, there exists a sequence $\left\{T_{j}\right\}$ of finitely continuous, bounded mappings, each satisfying condition $(S)_{+}$, which converges to $T$ uniformly on every bounded subset of $V$.

(b) If $T$ satisfies condition $(S)_{+}$, then $T$ is $A$-proper in the following sense [9], [10]: If $B$ is a closed ball of $V,\left\{V_{j}\right\}$ an increasing sequence of finite dimensional subspaces of $V$ whose union is dense in $V$, and if for each $j, u_{j}$ is an element of $V_{j} \cap B$ such that for a given element w of $V^{*}$,

$$
\left\|\phi_{j}^{*} T u_{j}-\phi_{j}^{*} w\right\|_{v_{j}^{*} \rightarrow 0} \quad(j \rightarrow \infty),
$$

where $\phi_{j}$ is the injection map of $V_{j}$ into $V, \phi_{j}^{*}$ the projection map of $V^{*}$ onto $V_{j}^{*}$, then there exists an infinite subsequence $\left\{u_{j(k)}\right\}$ converging strongly to an element $u$ of $B$ such that $T(u)=w$.

The proof of Theorem 4 is given in Chapter 17 of Browder [8]. The second property tells us that the generalized degree theory of Browder-Petryshyn [10] applies to mappings $T$ satisfying the condition $(S)_{+}$(for the details of this application, see[8]). The corresponding generalized degree theory for pseudomonotone maps follows from the convexity of the class of $T$ satisfying $(S)_{+}$and the following theorem whose proof will be published elsewhere:

Theorem 5. Let $X$ and $Y$ be Banach spaces, $G$ a bounded open subset of $X$, and consider an oriented approximation scheme $\left\{\left(X_{n}, Y_{n}\right.\right.$, $\left.\left.P_{n}, Q_{n}\right)\right\}$ for mappings $T$ of $\operatorname{cl}(G)$ into $Y$ in the sense of [10]. Let $Z$ be a convex family of $A$-proper mappings from $\mathrm{cl}(G)$ to $Y$ with respect to the given approximation scheme. Let $T$ be a mapping from $\operatorname{cl}(G)$ to $Y$ which is the uniform limit on $\mathrm{cl}(G)$ of mappings $T_{j}$ from the class $Z$. Then:

(a) For any sequence $\left\{T_{j}\right\}$ from $Z$ converging to $T$, if w does not lie in $\operatorname{cl}(T(\operatorname{bdry}(G)))$, then $\operatorname{Deg}\left(T_{j}, G, w\right)$ is the same for all $j$ sufficiently large and does not depend upon the choice of $\left\{T_{j}\right\}$. We denote this limit as $\operatorname{Deg}(T, G, w)$.

(b) $\operatorname{Deg}(T, G, w)$ is invariant under homotopy and weakly additive in the sense of Theorem 1 of $[10]$. If $\operatorname{Deg}(T, G, w) \neq\{0\}$ and if $T(\operatorname{cl}(G))$ is closed in $Y$, then w lies in $T(\mathrm{cl}(G))$.

(c) If $T$ is odd in the sense of Theorem 1 of $[10]$, then $\operatorname{Deg}(T, G, 0)$ consists only of odd integers, and $\operatorname{Deg}(T, G, 0) \neq\{0\}$.

ADDED IN PROOF. Results closely related to Theorem 5 have also been obtained by P. M. Fitzpatrick in connection with his Rutgers Ph.D. dissertation. 


\section{BIBLIOGRAPHY}

1. H. Brézis, Équations et inéquations non linéaires dans les espaces vectoriels en dualité, Ann. Inst. Fourier Grenoble 18 (1968), 115-175.

2. F. E. Browder, Nonlinear elliptic boundary value problems, Bull. Amer. Math. Soc. 69 (1963), 862-874. MR 27 \#6048.

3. - Nonlinear elliptic boundary value problems. II, Trans. Amer. Math. Soc. 117 (1965) 530-550. MR 30 \#4054.

4. - Fixed-point theorems for noncompact mappings in Hilbert space, Proc. Nat. Acad. Sci. U.S.A. 53 (1965), 1272-1276. MR 31 \#2582.

5. - Mapping theorems for noncompact nonlinear operators in Banach spaces, Proc. Nat. Acad. Sci. U.S.A. 54 (1965), 337-342. MR 31 \#5113.

6. - Nonlinear eigenvalue problems and Galerkin approximations, Bull. Amer. Math. Soc. 74 (1968), 651-656. MR 37 \#2043.

7. - Existence theorems for nonlinear partial differential equations, Proc. Sympos. Pure Math., vol. 16, Amer. Math. Soc., Providence, R. I. (to appear).

8. - Nonlinear operators and nonlinear equations of evolution, Proc. Sympos. Pure Math., vol. 18, Amer. Math. Soc., Providence, R. I. (to appear).

9. F. E. Browder and W. V. Petryshyn, The topological degree and Galerkin approximations for noncompact operators in Banach spaces, Bull. Amer. Math. Soc. 74 (1968), 641-646. MR 37 \#4678.

10. - Approximation methods and the generalized topological degree for nonlinear mappings in Banach spaces, J. Functional Analysis 3 (1969), 217-245.

11. J. Leray and J. L. Lions, Quelques résultats de Visik sur les problèmes elliptiques nonlinéaires par les méthodes de Minty-Browder, Bull. Soc. Math. France 93 (1965), 97-107. MR 33 \#2939.

12. G. J. Minty, On a "monotonicity" method for the solution of nonlinear equations in Banach spaces, Proc. Nat. Acad. Sci. U.S.A. 50 (1963), 1038-1041. MR 28 \#5358.

13. J. Neças, Sur l'alternative de Fredholm pour les opérateurs non-linéaires avec applications aux problèmes aux limites, Ann. Scuola Norm. Sup. Pisa (3) 23 (1969), $332-345$.

14. S. I. Pohožaev, The solvability of nonlinear equations with odd operators, Funkcional. Anal. i Priložen. 1 (1967), no. 3, 66-73. (Russian) MR 36 \#396.

15. M. I. Višik, Quasi-linear strongly elliptic systems of differential equations in divergence form, Trudy Moskov. Mat. Obšč. 12 (1963), 125-184=Trans. Moscow Math. Soc. 1963, 140-208. MR 27 \#6017.

University of Chicago, Chicago, Illinois 60637 